genetic features (POLE mutation [POLEmt], microsatelliteinstability high [MSI-H], homologous recombination defect [HRD], MUC16 mutation [MUC16mt]) showed significant overlap. In Kaplan-Meier survival analyses, MIS and open surgery brought similar survival outcome in patients with POLEmt, MSI-H, HRD or MUC16mt. But in POLE wild type, non MSI-H, non HRD, or MUC16 wild type patients, MIS resulted in shorter recurrence-free survival (RFS) $(p=0.008,0.015,0.003,0.008)$. Based on TCGA classification, POLE ultramutated and MSI hypermutated type had similar prognosis after two surgeries, while copy-number low type without CTNNB1 mutation and copy-number high type with TP53 mutation showed more rapid recurrence after MIS $(p=0.048$ and 0.037$)$. Further analyses were done to simplify the model. In patients with $\geq 1$ of the 4 features (POLEmt, MSI-H, HRD or MUC16mt), MIS and open surgery brought comparable overall survival and RFS $(p=0.339$ and 0.969); for patients with none of the features, especially those with wild type CTNNB1 or TP53 mutation, longer RFS was observed in open surgery group $(\mathrm{p}=0.001,<0.001,<0.001$, respectively). All the results of Kaplan-Meier analyses were verified by Cox regressions.

Conclusion The molecular features of EC are related to patients' prognosis after different surgical approaches. MIS should be recommended in patients with POLEmt, MSI-H, HRD or MUC16mt for similar survival outcome and less perioperative complications compared to open surgery.

Disclosures This work was supported by the National Natural Science Foundation of China (81972426, 81202041, 81672571, 81874108 and 81802607), Special Projects for Strengthening Basic Research of Peking University (BMU2018JC005), National Key Technology R\&D Program of China (2019YFC1005200 and 2019YFC1005201).

The authors have no potential conflict of interest to disclose.

\section{THE POSITIVE PELVIC LYMPH NODES IN ENDOMETRIAL CANCER - HISTOPATHOLOGICAL PARAMETERS AS PREDICTORS}

${ }^{1}$ Aljosa Mandic, ${ }^{2}$ Dunja Kokanov, ${ }^{3}$ Bojana Gutic, ${ }^{3}$ Tatjana Ivkovic Kapicl, ${ }^{3}$ Slobodan Maricic, ${ }^{3}$ Nenad Solajic. 'University of Novi Sad; Medical Faculty; Oncology Institute of Vojvodina; ${ }^{2}$ Oncology Institute of Vojvodina; Oncology Institute of Vojvodina; ${ }^{3}$ University of Novi Sad; Medical Faculty; Oncology Institute of Vojvodina

\subsection{6/ijgc-2020-ESG0.57}

Introduction/Background Endometrial cancer is the most common malignancy of the female reproductive tract. Lymph node metastases are an important prognostic factor in endometrial cancer. Several prognostic factors have been shown to correlate with lymph node metastasis: depth of myometrial invasion, cervical infiltration, histologic grade of the tumor, tumor diameter, serous histology, lymphovascular invasion, and positive peritoneal cytology.

Methodology Finding the pathohistological parameters that will indicate with greater certainty the possibility of metastases in the lymph nodes, on the basis of which it will be evaluated whether such patients should undergo lymphadenectomy or not. A retrospective analysis of patients with endometrial cancer who underwent surgery at the Oncology Institute of Vojvodina (Clinic for Operative Oncology - Department of Gynecology) in the period from 2012 to 2018. The study included 120 patients who underwent hysterectomy with bilateral adnexectomy and pelvic lymphadenectomy.

Results Among patients who had lymph node metastases, there were statistically significant more patients $(p<0.01)$ with endometrial cancer of histological type 2, with depth of myometrial invasion greater than 50\%, cervical stroma infiltration, lymphovascular invasion, and positive peritoneal cytology.

Conclusion Histopathological parameters such as type 2 endometrial cancer, myometrial invasion depth greater than 50\%, cervical stroma infiltration, lymphovascular invasion and positive peritoneal cytology increase the chances of lymph node metastases. Tumor size $(>2 \mathrm{~cm})$ as well as histologic grade did not correlate with a higher incidence of lymph node metastases. In this study, both the parametrial infiltration and the number of lymph nodes removed have clinical significance, but not statistical significance.

\section{AN AUDIT OF THE USE OF VAGINAL BRACHYTHERAPY IN ENDOMETRIAL CANCERS}

Bhargavi Ilangovan, Ganapathy Raman, S Ayyappan. Madras Cancer Care Foundation

\subsection{6/ijgc-2020-ESG0.58}

Introduction/Background Vaginal Brachytherapy is an effective treatment modality to prevent local recurrence in endometrial cancers. We did an audit of the Endometrial cancer cases treated by an oncology group practice over a period of 5 years to assess the use of VBT.

Methodology The data of 106 patients treated for endometrial cancers between 2014 and 2019 by an oncology group across 2 tertiary care centres was retrospectively analysed.

The indications of vaginal brachytherapy, number of fractions, dose per fraction, prescription points, vaginal stenosis on follow up and use of vaginal dilators were the variables collected and were entered in an excel sheet.

Results A total of 106 patient data was entered and analysed. The mean age of the patients was 60 years. The radiotherapy details were available for 84 patients. Of the 84 patients analysed, 59 patients (70\%) received adjuvant Vaginal Brachytherapy, while $25(30 \%)$ did not. Of the patients who received VBT, $32(54.2 \%)$ patients received VBT as a boost after Pelvic RT (figure 1).

VBT BOOST: Among the 32 patients who received vaginal Brachytherapy as a boost after Pelvic RT, only $4(12.5 \%)$ patients had cervix involvement. Lower uterine segment was involved in 12 patients.

The EBRT dose was $45-50 \mathrm{~Gy}$ in 25 to 28 fractions.

Abstract 165 Table 1 The stagewise and grade wise numbers

\begin{tabular}{lc}
\hline STAGE & NUMBER \\
\hline $\mathrm{IA}, \mathrm{G} 1$ & 6 \\
$\mathrm{IA}, \mathrm{G} 2$ & 3 \\
$\mathrm{IA}, \mathrm{G} 3$ & 5 \\
$\mathrm{IB}, \mathrm{G} 1$ & 4 \\
$\mathrm{IB}, \mathrm{G} 2$ & 3 \\
$\mathrm{II}$ & 2 \\
$\mathrm{III} A$ & 2 \\
\hline
\end{tabular}

